\title{
Age-Related Changes in the Ability to Switch between Temporal and Spatial Attention
}

\author{
Eleanor Callaghan ${ }^{1,2}$, Carol Holland ${ }^{1}$ and Klaus Kessler ${ }^{2 *}$ \\ ${ }^{1}$ Aston Research Centre for Healthy Ageing, Aston University, Birmingham, UK, ${ }^{2}$ Aston Brain Centre, Aston University, \\ Birmingham, UK
}

Background: Identifying age-related changes in cognition that contribute towards reduced driving performance is important for the development of interventions to improve older adults' driving and prolong the time that they can continue to drive. While driving, one is often required to switch from attending to events changing in time, to distribute attention spatially. Although there is extensive research into both spatial attention and temporal attention and how these change with age, the literature on switching between these modalities of attention is limited within any age group.

Methods: Age groups (21-30, 40-49, 50-59, 60-69 and 70+ years) were compared on their ability to switch between detecting a target in a rapid serial visual presentation (RSVP) stream and detecting a target in a visual search display. To manipulate the cost of switching, the target in the RSVP stream was either the first item in the stream (Target 1st), towards the end of the stream (Target Mid), or absent from the stream (Distractor Only). Visual search response times and accuracy were recorded. Target

OPEN ACCESS

Edited by:

Thomas Espeseth, University of Oslo, Norway

Reviewed by: Lakshmi Rajagopal, Northwestern University, USA Inge Linda Wilms, University of Copenhagen, Denmark Derrick G. Watson, University of Warwick, UK

${ }^{*}$ Correspondence: Klaus Kessler k.kessler@aston.ac.uk

Received: 14 September 2016 Accepted: 30 January 2017 Published: 14 February 2017

Citation:

Callaghan E, Holland C and Kessler K (2017) Age-Related Changes in the Ability to Switch between Temporal and Spatial Attention. Front. Aging Neurosci. 9:28. doi: 10.3389/fnagi.2017.00028 1st trials behaved as no-switch trials, as attending to the remaining stream was not necessary. Target Mid and Distractor Only trials behaved as switch trials, as attending to the stream to the end was required.

Results: Visual search response times (RTs) were longer on "Target Mid" and "Distractor Only" trials in comparison to "Target 1st" trials, reflecting switch-costs. Larger switchcosts were found in both the 40-49 and 60-69 years group in comparison to the 21-30 years group when switching from the Target Mid condition.

Discussion: Findings warrant further exploration as to whether there are age-related changes in the ability to switch between these modalities of attention while driving. If older adults display poor performance when switching between temporal and spatial attention while driving, then the development of an intervention to preserve and improve this ability would be beneficial.

Keywords: spatial attention, temporal attention, aging, switching, cognitive flexibility

\section{BACKGROUND}

Driving cessation can be detrimental to older adults' independence and has been shown to be a risk factor in developing depression (Marottoli et al., 1997; Ragland et al., 2005; Windsor et al., 2007). Identifying age-related changes in cognition that contribute towards reduced driving performance is the first step in a trajectory of research towards developing an 
intervention to improve older adults' driving. This could lead to long-term advantages such as prolonging the time that people can continue to drive and help to preserve their independence.

At-fault collision statistics show that, while older adults have an overall reduced crash risk in comparison to young drivers, they present a disproportionate risk of at-fault collisions at intersections and collisions caused by a failure to give way, or to notice other objects, stop signs or signals (HakamiesBlomqvist, 1993; McGwin and Brown, 1999; Guo et al., 2010; Arai and Arai, 2015). Consistent with higher risks at intersections (Hakamies-Blomqvist, 1993), in their seminal work Parasuraman and Nestor (1991) concluded that older drivers' accidents were often due to failures in attention, particularly selective attention and switching. These findings are consistent with older drivers' own self-perceptions, who have reported an increased difficulty to read and process signs in time (Musselwhite and Haddad, 2010). It is therefore a viable hypothesis that changes in spatial attention and attention switching are having an impact on driving skills later in life.

\section{Spatial Attention}

There is extensive research demonstrating the relationship between spatial attention and driving performance and exploring how this changes with age (Hennessy, 1995; Richardson and Marottoli, 2003; Hoffman et al., 2005; Ball et al., 2006; Leversen et al., 2013; Cuenen et al., 2015). However, poor spatial attention does not result in poor driving in all older individuals (Vaucher et al., 2014). These findings highlight the need to further investigate attentional deficits in older drivers and identify the factors that determine whether deficits in attention affect driving performance.

There is a consensus that there is no specific decline in visual search performance with healthy aging when the target is distinct from the distractors and "pops out" of the display-i.e., a pop-out search (Plude and Doussardroosevelt, 1989; Foster et al., 1995; Humphrey and Kramer, 1997; Bennett et al., 2012; Li et al., 2013). Although older adults show increased response times (RTs) to detect targets in pop-out searches in comparison to young adults, age group differences in RTs remain constant with increasing numbers of distractors (Plude and Doussardroosevelt, 1989) and have therefore been attributed to general slowing (Foster et al., 1995). In contrast, visual search performance is thought to decline with age when the target is visually indistinct from distractors (i.e., in so-called "conjunction search", where targets are defined as a combination of features shared with the distractors) and a serial search is required (Plude and Doussardroosevelt, 1989; Foster et al., 1995; Humphrey and Kramer, 1997; Bennett et al., 2012; Li et al., 2013). The increase in RTs with increasing numbers of distractors gets steeper with age, suggesting a specific deficit in serial visual search rather than a general slowing of RTs.

It is often argued that older adults have deficits in inhibiting irrelevant visual information (Hasher and Zacks, 1988; Greenwood and Parasuraman, 1994; Adamo et al., 2003; Maciokas and Crognale, 2003; Lustig et al., 2007; Gazzaley et al., 2008). It may be that poor selective attention is caused by deficits in inhibitory mechanisms. Competition models of visual selective attention (Treisman, 1988; Desimone, 1998; Bundesen et al., 2005; Beck and Kastner, 2009; Scalf et al., 2013) and evidence from single cell recordings (Reynolds et al., 1999) suggest that multiple stimuli are processed in parallel but in separate cell groups (Luck et al., 1997). Visual stimuli compete for processing resources and attention is implemented to bias excitation in favor of salient and task relevant stimuli. The Neural Theory of Visual Attention (NTVA; Bundesen et al., 2005) proposes that attention works to increase or decrease the number of cells involved in processing each object and alters the firing rate of neurons coding for certain features. Impairments in these excitatory-inhibitory attentional mechanisms may lead to difficulties in inhibiting irrelevant visual information and exciting target stimuli in older adults. This hypothesis would explain older participants' disproportionately increased number and duration of saccadic eye movements on serial visual searches (Porter et al., 2010). In contrast, Lien et al. (2011) demonstrated that older and younger participants were equally able to attend to task-relevant stimuli and inhibit salient but irrelevant stimuli. However, it may be that the salience of the distractors aided inhibition due to the distinct visual features prompting a strong inhibitory response. Thus, deficits in excitatory-inhibitory mechanisms could lead to difficulties in selective attention.

There is evidence to suggest that older adults compensate for excitatory-inhibitory deficits with top-down control of attention. Neider and Kramer (2011) found that older participants not only benefited more than younger participants from using contextual information in a visual search within a realistic scene, but also displayed greater costs to their performance when the location of the visual search target was incongruent with its contextual information. Furthermore, McLaughlin and Murtha (2010) found that older adults utilized cues more than younger people in a visual search task. Similarly, Watson and Maylor (2002) demonstrated that the benefits of visual marking were preserved in adults aged $65-80$ years. Visual marking is where a proportion of distractors within a visual search task is shown before the onset of the remaining distractor stimuli and the target stimulus, enabling top-down driven inhibition of the distractors that were presented first. However, the benefits of visual marking were not preserved in older adults when visual search items were moving. Previous research has demonstrated that older participants have lower motion detection thresholds (Conlon and Herkes, 2008) and find it more difficult to judge the speed of moving stimuli and vehicles (Scialfa et al., 1991; Schiff et al., 1992; Norman et al., 2003; Snowden and Kavanagh, 2006). The absence of visual marking of moving stimuli in older adults could therefore be due to difficulties in processing moving stimuli. Together findings suggest that older participants may rely more on top-down processes to compensate for declines in excitatory-inhibitory mechanisms in attention.

\section{Temporal Attention}

In addition to the importance of spatial attention in driving, the allocation of attention to events changing in time, i.e., temporal attention, is important to be able to attend, process and respond to rapidly changing visual stimuli in a dynamic environment 
such as driving. It is well established that older adults require longer to process visual stimuli-i.e., have slower processing speeds (Ball et al., 2006; Rubin et al., 2007) and display an increased magnitude of the so-called "attentional blink" (Lahar et al., 2001; Maciokas and Crognale, 2003; Lee and Hsieh, 2009; Shih, 2009; van Leeuwen et al., 2009). The attentional blink is the reduced ability to detect a second target (T2) in a rapidly changing stream of stimuli-i.e., a rapid serial visual presentation (RSVP) stream-up to $500 \mathrm{~ms}$ after detecting a first target (T1) in the stream (Raymond et al., 1992). This effect is inflated and lasts for an increasing length of time with increased age. There is evidence to suggest that, whereas individuals in their 60s have no difficulties in temporal attention (Lee and Hsieh, 2009; Quigley et al., 2012), difficulties may begin to develop between the ages of 70-80 years (Conlon and Herkes, 2008; Shih, 2009). Conlon and Herkes (2008) concluded that impairments were due to slowed processing speed. However, age-related deficits observed in other temporal attention tasks have been demonstrated not to be due to general slowing (Maciokas and Crognale, 2003; Lee and Hsieh, 2009). Difficulties in temporal attention in those aged over 70 years could therefore be due to a specific decline in selective attentional mechanisms and could share the same underlying cause as difficulties in spatial selective attention, i.e., in excitatory-inhibitory selective attention processes, where excitatory mechanisms fail to respond to the target and inhibitory mechanisms fail to mitigate interference from the distractors in the RSVP stream.

\section{Switching Attention between Time and Space}

Equally vital to safe driving, particularly at intersections, is the ability to switch between temporal and spatial attention. For example, when driving, one must switch from attending to fast moving and changing cars on the road ahead, to distributing attention across space to attend to road signs and surrounding hazards. Although there is extensive research displaying inflated switch-costs with increased age in task switching paradigms (Cepeda et al., 2001; Gamboz et al., 2009; Gold et al., 2010) there has been very little research on switching between different modalities of attention in any age group.

Overlapping networks across occipital, frontal, parietal and motor regions have been implicated in both directing attention in time and space (Coull and Nobre, 1998; Shapiro et al., 2002; Gross et al., 2004; Fu et al., 2005; Madden et al., 2007; Li et al., 2013). Although Coull and Nobre (1998) found overlapping activation for both temporal and spatial attention in a functional magnetic resonance imaging (fMRI) study, they found that patterns of activation for the two types of attention were distinct. In an extension of the NTVA, it has been proposed that as temporal expectation increases temporal attention works to increase the firing rate of neurons that represent certain features. In contrast, one would expect spatial attention to alter the number of cells allocated to processing objects in the visual field (Bundesen et al., 2005; Vangkilde et al., 2012, 2013). Thus, it may be expected that switching between temporal and spatial attention requires the efficient re-allocation of cells to receptive fields, as well as changes to the perceptual bias towards features which in turn influences the firing rate of neurons.

There is limited research into age-related changes in the ability to switch between temporal and spatial attention. Jefferies et al. (2015) demonstrated that younger adults require less time than older adults to narrow their focus of attention from two RSVP streams to one, indicating that there may be an age-related decline in the redistribution of attention spatially from a single location. However, both RSVP streams remained on the screen. Rather than a deficit in switching to distribute attention spatially, increased times taken to divert attention may be due to an age-related impairment in disengaging from task irrelevant stimuli (Greenwood and Parasuraman, 1994). In Lee and Hsieh (2009) study, participants switched from attending to an RSVP stream to identify a target, to allocating their attention in space to identify and point to a masked peripheral target in varying locations. Although the older age group displayed lower performance when the peripheral target was presented at 100,300 and $700 \mathrm{~ms}$ after the RSVP target onset, lower performance was exaggerated at 100 and $300 \mathrm{~ms}$. These findings show that older participants had greater difficulties in switching from temporal to spatial attention when there was $300 \mathrm{~ms}$ or less between target onsets. Russell et al. (2013) has since replicated these findings, further demonstrating that the impairment lasted for $450 \mathrm{~ms}$. However, Lee and Hsieh (2009) aim was to investigate the attentional blink in older adults, resulting in a failure to distinguish between impaired task performance resulting from an increased attentional blink after processing the RSVP target, or due to increased switch-costs between temporal and spatial attention. Poorer performance at 100 and $300 \mathrm{~ms}$, but not $700 \mathrm{~ms}$, could equally be due to requiring longer to switch between temporal and spatial attention, or an extended attentional blink. A comparison of the relevant attentional blink and attention switching literature is presented in Table 1. The table compares the duration of the attentional blink in older age groups in addition to the duration of impairment from attention switching.

\section{The Current Study}

The aim of the current study was to explore whether there are age-related changes in the ability to switch between temporal and spatial attention and to explore the cognitive mechanisms that might underpin these changes. Age groups were compared on their ability to switch from allocating attention in time, in order to identify a single target in an RSVP stream, to allocating attention spatially, in order to identify a visual search target. To manipulate the cost of switching, the position of the target in the RSVP stream was either the first item in the stream, towards the end of the stream, or absent from the stream. When the target was the first item in the stream (Target 1st condition), participants were no longer required to attend to the stream, and thus no cost of switching was expected. On the contrary, when the target was near the end of the stream (Target Mid condition) or the stream consisted of only distractor items (Distractor Only condition), participants needed to attend to the stream until towards the end of the stream, inducing a cost of switching. Longer visual search RTs were therefore expected 
TABLE 1 | Comparison of results from previous studies.

\begin{tabular}{llll}
\hline Author & $\begin{array}{l}\text { Mean age } \\
\text { (years) }\end{array}$ & Method & Duration of impairment \\
\hline Attentional blink & & & $520 \mathrm{~ms}$ \\
Lahar et al. (2001) & 68.70 & Attentional blink & $300 \mathrm{~ms}$. No impairment at 700 ms \\
$\begin{array}{l}\text { Lee and Hsieh (2009) } \\
\text { Maciokas and Crognale (2003) }\end{array}$ & 59.30 & Attentional blink & $824 \mathrm{~ms}$ \\
Switching & $64-79$ & Attentional blink & $266 \mathrm{~ms}$ \\
Jefferies et al. (2015) & 66.40 & Time taken to narrow focus from 2 to 1 RSVP stream & $300 \mathrm{~ms}$. No impairment at 700 ms \\
Lee and Hsieh (2009) & $55-62$ & Attention switch from temporal to spatial attention & $450 \mathrm{~ms}$ \\
Russell et al. (2013) & 66.00 & Attention switch from temporal to spatial attention & \\
\hline
\end{tabular}

when switching from the single target RSVP task to the visual search in both the Target Mid and Distractor Only conditions, which each behaved as switch conditions, in comparison to the Target 1st condition, which behaved as a no-switch condition. It was hypothesized that there would be an age-related increase in the cost of switching from the RSVP task to initiate the visual search task, which would be reflected in greater increases in RTs from the no-switch condition to the two switch conditions in the older groups in comparison to the younger groups. In contrast to Lee and Hsieh (2009) study, the inclusion of the Distractor Only condition enabled the investigation of whether any observed differences were due to difficulties in switching between attentional mechanisms or an increased attentional blink. If there is a deficit in switching between attentional mechanisms, then age-related inflated switch-costs would be present in both the Distractor Only and Target Mid conditions. Conversely, if age-related increases in switch-costs result from an extended attentional blink after processing the RSVP target, then age differences in switch-costs would only be observable in the Target Mid and not the Distractor Only condition.

Based on previous evidence that suggests that visual selective attention to temporal events is more difficult in those over the age of 70 years (Conlon and Herkes, 2008; Shih, 2009) but not in those aged 60-70 years (Lee and Hsieh, 2009; Quigley et al., 2012), it was expected that participants in the $70+$ years age group would detect and identify fewer targets in the RSVP stream in comparison to younger adults, but that the 60-69 years age group would not be impaired.

It is well established that there is a decline in working memory capacity with increased age (Richardson and Vecchi, 2002; Toepper et al., 2014). It could be argued that the increased working memory load from retaining the target digit in the current task could impair older participants' performance in switching. However, it is unlikely that retaining a single target would place enough demand on working memory to affect task performance. Furthermore, Akyürek and Hommel (2005) demonstrated that working memory load does not interact with the duration of the attentional blink, implying that working memory load should not affect visual search target processing. Although working memory capacity is unlikely to affect task performance in the current task, performance is likely to be affected by age-related declines to the central executive (Baddeley, 1992). Baddeley's (1992) working memory model proposed that the central executive controls the allocation of attentional resources. It may therefore be expected that a decline in executive function could affect the ability to switch from allocating attention to events changing in time (i.e., the RSVP stream) to distribute attention spatially (i.e., to visual search stimuli). We therefore implemented the Random Number Generation task (RNG) to measure executive functions of updating and inhibition (Miyake et al., 2000) in order to examine the effect of executive function on task performance. Performance on random generation tasks has previously been found to decline with age (van der Linden et al., 1998).

Consistent with age-related declines in serial but not pop-out search performance (Plude and Doussardroosevelt, 1989; Foster et al., 1995; Humphrey and Kramer, 1997; Bennett et al., 2012; Li et al., 2013) and general slowing of RTs (Salthouse, 2000; Verhaeghen and Cerella, 2002), it was predicted that there would be an age-related increase in visual search RTs that would be greater for serial than pop-out searches.

To establish an understanding of the mechanisms that underpin switching between modalities of attention, additional cognitive measures were recorded. The useful field of view (UFOV) task was implemented to measure visual processing speed, divided attention and selective attention. Performance on the UFOV (Ball et al., 2006; Rubin et al., 2007; Edwards et al., 2009) tasks have been found to decline with age.

\section{MATERIALS AND METHODS}

\section{Participants}

One hundred and five participants in five age groups (21-30, $40-49, \quad 50-59, \quad 60-69$, and $70+$ years) participated. The 21-30 years group were used as a comparison group for age-related cognitive changes for all other groups and the 40-49 and 50-59 years groups were used as middle-aged comparison groups for the 60-69 and 70+ years groups. Due to the study being advertized as research related to driving, many of the participants were regular drivers. The percentage of participants in each group who could drive are displayed next to participant demographics in Table 2. Participants with photosensitive epilepsy were excluded from participation, in addition to those who scored less than the 87 cut off for possible cognitive impairment on the Addenbrookes Cognitive Examination 3 (ACE-3; Noone, 2015). The ACE-3 consists of a series of short tasks which provide measures of language, memory, attention, fluency and visuospatial abilities. 


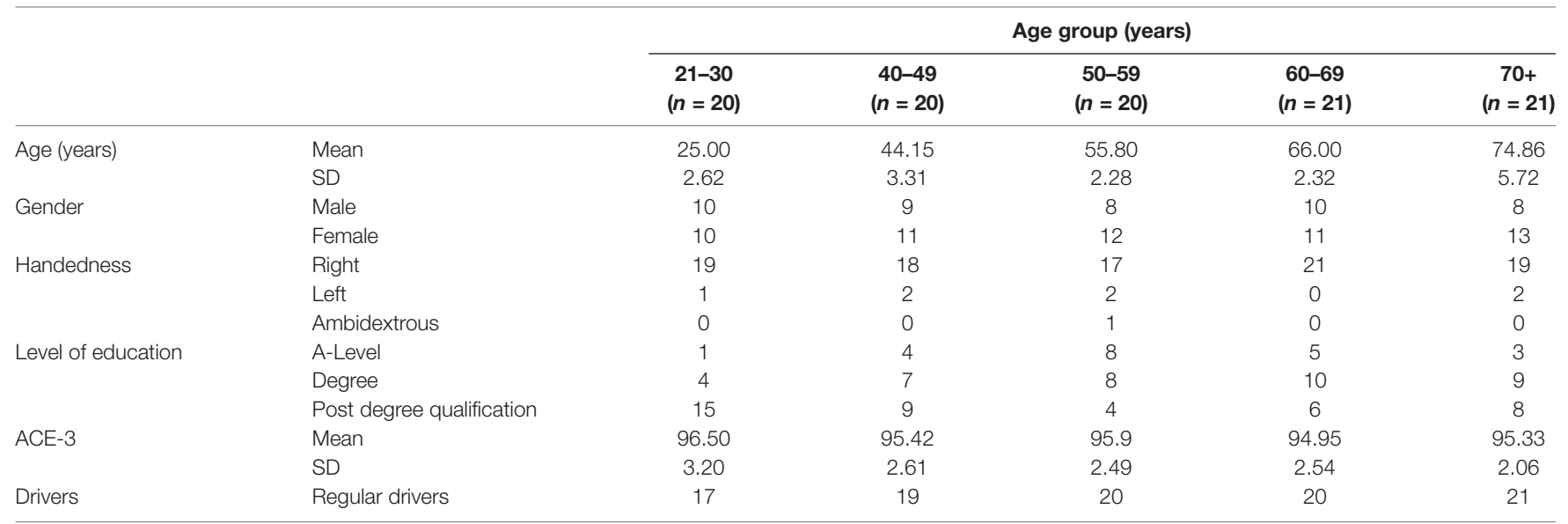

The number of participants who are left and right handed, and the number of participants who are male and female are presented for each age group, in addition to the mean age of each age group. One participant from the 40-49 years group was excluded from the ACE-3 analysis as their performance was impaired on vocabulary dependent sections of the task due to English being their second language.

Participants in the $21-30$ and $40-49$ years groups were recruited from Aston University staff and students and the community. Participants aged over 60 years were recruited from the Aston Research Centre for Healthy Ageing participation panel and University of the Third Age groups around the West Midlands. Participants received $£ 7.50$ towards their travel expenses. All participants provided written informed consent before participating. The research was approved by Aston University Research Ethics Committee. Vulnerable populations were not involved.

One participant from the 60-69 years group and two from the 70+ years group scored equal to or lower than the 87 cut-off on the ACE-3 (Noone, 2015) and were therefore excluded from further analyses. One participant in the 40-49 years group scored lower than 87 on the ACE-3, however, this was due to English being their second language and so they were not excluded from the study. Their ACE-3 score was excluded from the analysis. All other participants scored over 87. After excluding participants with low ACE-3 scores, the mean age of the 60-69 years group was 66.00 years $(S D=2.32)$, and the mean age for the $70+$ years groups was 74.86 years $(S D=5.72)$.

Fisher's Exact test comparing group differences in level of education (A-level equivalent or lower/degree equivalent/post degree qualification) revealed a significant difference in the level of education between groups $(p=0.049)$. The number of participants in the 21-30 years group with post degree qualifications was greater than expected $(z=2.1)$. A one-way ANOVA comparing group differences in general cognitive function measured with the ACE-3 revealed no significant group differences in ACE-3 scores ( $p>0.05)$.

\section{Materials and Procedures}

\section{Attention Switching Task}

In the attention switching task, participants alternated between attending to an RSVP stream and attending to a visual search display. Each trial consisted of a fixation cross, presented for $2000 \mathrm{~ms}$, followed by the RSVP stream, which was immediately followed by the visual search display. Stimuli were presented on stimulus presentation software E-Prime 2.0 Professional (Psychology Software Tool. Inc., Sharsburg, PA, USA) on a windows computer, on a $22^{\prime \prime}$ monitor $(1280 \times 1050$ resolution $)$ which was approximately $55 \mathrm{~cm}$ in front of the participant. All stimuli were presented in black (RGB $0-0-0$ ) on a gray background (RGB 192-192-192).

The RSVP stream consisted of a rapidly changing stream of letters in the center of the display. There were ten items in each RSVP stream, each presented for $100 \mathrm{~ms}$ with no inter-stimulus interval. Stimuli were presented in font size $30 \mathrm{pt}(0.75 \times 0.75 \mathrm{~cm}$, $\left.0.78^{\circ}\right)$. On two thirds of the trials, one of the items in the stream was a target digit ranging from 1 to 9 . The participant's task was to remember the digit. The remaining one third of the trials contained no target. Based on their visual similarity to certain numbers, letters I, O and S were excluded from the stream, as well as visual search targets $\mathrm{K}$ and $\mathrm{Z}$. It should be noted that the current RSVP task differs from the attentional blink paradigm as the RSVP stream contains only a single target.

The visual search display consisted of eight letters presented in a circle around a fixation cross in the center of the screen, including seven distractors and one target. The target letter was always either a " $K$ " or a " $Z$ ". Stimuli were presented in font size $20 \mathrm{pt}\left(0.50 \times 0.50 \mathrm{~cm}, 0.52^{\circ}\right)$ and the center of each stimulus was $2.3 \mathrm{~cm}\left(2.40^{\circ}\right)$ from the center of the fixation cross. Participants pressed the "space-bar" once they identified the target. Participants' RTs to press the space-bar were recorded. An initial space-bar response was implemented instead of a choice RT to minimize variability in RTs that result from the additional process of deciding which key to press. In a pilot study we found that while the overall pattern of means was the same, there was increased variability in RTs when participants responded by pressing either a " $K$ " or " $Z$ " key, depending on which letter the visual search target was, in comparison to using a single space-bar response. Higher variability from a choice RT may have affected older more than younger participants' performance. Older participants are 
thought to show increased variability in RTs (Hultsch et al., 2002) and so it is important to minimize this variability. Participants were instructed to keep their eyes fixed on the cross while they completed the visual search and to respond as quickly as possible. Participants then indicated by typing on the keyboard whether it was a " $K$ " or a " $Z$ " in the display, followed by whether they had seen a target digit in the RSVP stream by typing " $\mathrm{Y}$ " if they had, and " $N$ " if they had not. If a digit was correctly detected in the RSVP stream, participants then typed on the keyboard which number they saw. Accuracy throughout the task was recorded. Participants wore headphones through which a "ding" sound was played after a correct response and a chord sound was played after an incorrect response.

On $50 \%$ of the trials the visual search display was a "popout" visual search, in which the distractors were all the letter " $\mathrm{P}$ ", allowing the target to "pop-out" to the participant. On $50 \%$ of the trials the visual search display was a "serial" visual search, in which all distractor letters were unique prompting a serial search. To manipulate the cost of switching, the position of the target in the RSVP stream that preceded the visual search was either the first item in the stream (Target 1st), which behaved as a no-switch condition, or the target was either the seventh, eighth or ninth item in the stream (Target Mid) or absent from the stream (Distractor Only), which both behaved as switch conditions. Illustrations of the RSVP stream and of the visual search display are presented in Figure 1.

There were 30 trials of each of the six conditions (Pop-out search: Target 1st/Target Mid/Distractor Only; Serial search: Target 1st/Target Mid/Distractor Only), with a total of 180 trials. To provide the opportunity for breaks, trials were divided into 10 blocks. Trials were randomized within blocks. Participants completed 10 practice trials before starting the experimental trials. One participant from the 40-49 years group, one from the 50-59 years group, five from the 60-69 years group and 11 from the $70+$ years group required an additional 10 practice trials.

\section{Useful Field of View Task}

The Useful Field of View task (UFOV; Ball et al., 1988) was administered to measure processing speed, selective attention and divided attention. The UFOV consists of three sub-tasks on the computer, where the stimulus presentation duration begins at $500 \mathrm{~ms}$ and reduces to $16.7 \mathrm{~ms}$ until the participant achieves less than $75 \%$ accuracy. The shortest presentation duration at which the participant achieves $75 \%$ accuracy is recorded as the participant's processing speed threshold in each of the tasks.

On the processing speed task, either a picture of a car or a picture of a truck was presented in the center of the screen. Participants then indicated whether the image presented to them was a car or a truck. The divided attention task was the same as the processing speed task with the addition of the simultaneous presentation of a peripheral stimulus, which was also either a car or a truck. Participants both identified the item presented in the center of the screen and the location of the peripheral stimulus. The selective attention task was the same as the divided attention task with the addition of distractor stimuli simultaneously presented surrounding the two target stimuli. A full description of the UFOV has been described previously by Ball et al. (1993).

\section{Random Number Generation task}

The RNG (Towes and Neil, 1998) was administered to measure executive functions. For $2 \mathrm{~min}$, participants were played a

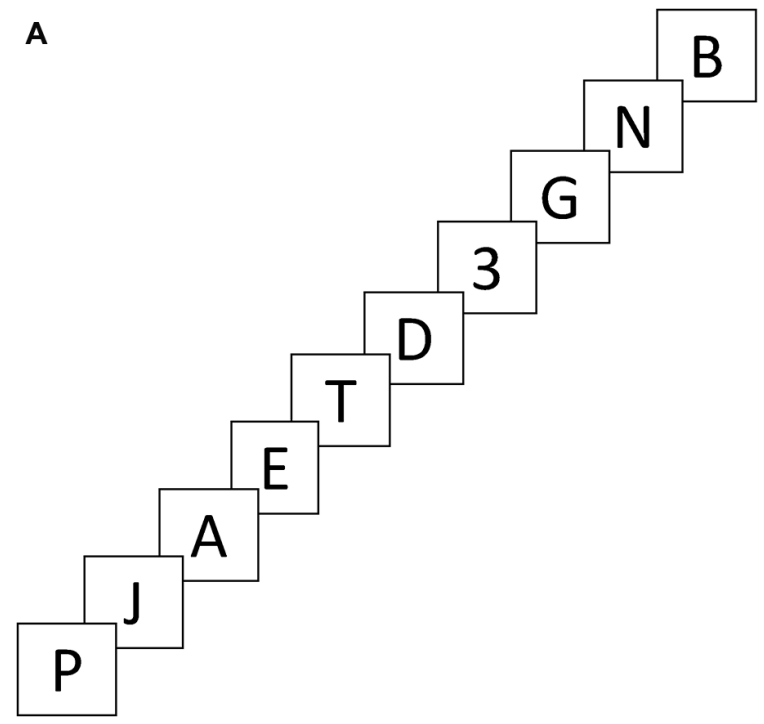

B

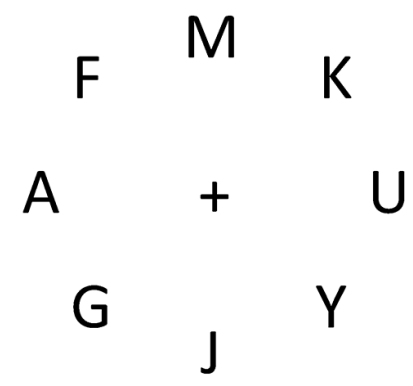

FIGURE 1 | Illustration of examples of the experiment set up. The rapid serial visual presentation (RSVP) stream illustration (A) displays a Target Mid RSVP stream, and the visual search display illustration (B) displays a serial visual search display. Each trial consisted of a fixation cross (2000 ms) followed by an RSVP stream immediately followed by a visual search display. 
metronome beat at 60 beats per minute and called aloud random numbers from 1 to 9 in time with the beat. Random was defined using Horne et al.'s (1982) hat analogy.

Towes and Neil (1998) software, Rgcalc, was used to calculate measures of randomness. In accordance with Miyake et al. (2000) Principal Components Analysis, Evans' (1978) RNG score, a measure of how frequently number pairs/triplets occurred, was selected to measure inhibition, and Redundancy (R), a measure of how frequently each number occurred, was selected to measure updating. Lower scores on each measure indicates poorer randomization.

\section{Data Analysis}

Data were analyzed using Statistical Package for Social Sciences (SPSS 21).

\section{Attention Switching Task}

Participants' median visual search RTs (ms) on trials where responses were correct on both the visual search and RSVP tasks were extracted using E-Prime data viewing application E-DataAid. Participants' proportions of correct visual search target identifications, RSVP target detections, RSVP target identifications and correct responses on the RSVP task on the Distractor Only condition were also extracted.

Older adults display higher proportions of false positive responses than younger individuals (Kenemans et al., 1995; Bolton and Staines, 2012). D prime ( $\left.\mathrm{D}^{\prime}\right)$ was used as an uniased measure of RSVP target detection sensitivity, as has been done in previous work on visual attention in the aging population (Parasuraman et al., 1989; Mouloua and Parasuraman, 1995; Berardi et al., 2001). Differences between age groups and RSVP conditions in both RSVP target detection and target identification were analyzed in two $2 \times 5$ mixed ANOVA, with RSVP condition (Target Mid/Target 1st) as a within subjects factor, and age group (21-30/40-49/50-59/60-69/70+ years) as a between subjects factor.

Differences in median visual search RTs between age groups, visual search conditions and RSVP conditions were analyzed in a $2 \times 3 \times 5$ mixed ANOVA, where within subject factors were visual search condition (serial/pop-out) and RSVP condition (Distractor Only/Target Mid/Target 1st), and age group (21-30, $40-49,50-59,60-69,70+$ years) was a between subjects factor. Multiple comparisons were corrected for with Bonferroni correction.

The data were expected to violate assumptions of equality of variance due to increases in inter-individual variability with age (Hale et al., 1988; Morse, 1993). There is evidence to support that the ANOVA is robust to violations to homogeneity of variance (Budescu and Appelbaum, 1981; Budescu, 1982). Levene's test for equality of variance is therefore not reported.

To further explore the interactions between independent variables that were identified from the ANOVA on RSVP accuracy, independent $t$-tests were implemented to compare age groups on target identification separately for Target Mid and Target 1st RSVP conditions.

To further explore the interactions between independent variables that were identified in the RT ANOVAs, percentage differences between conditions were calculated for each individual and independent $t$-tests were implemented to compare age groups' percentage differences in RTs. It is important to note that $t$-tests were exploratory rather than hypothesis driven, and hence Restricted Fisher's Least Significant Difference test was applied and corrections for multiple comparisons were not conducted (Snedecor and Cochran, 1967). Where Levene's test for equality in variance was significant $(p<0.05)$ when computing $t$-tests, "Equality of variance not assumed" statistics were reported.

\section{Cognitive Measures}

The relationship between switch-costs and each cognitive measure, including UFOV subtasks processing speed, divided attention and selective attention and RNG indices for updating (R) and inhibition (RNG), and the relationship between each UFOV subtask and pop-out search RTs on the Target 1st condition, were explored with Spearman's correlation analyses. It should be noted that correlations were exploratory and corrections for multiple comparisons were not conducted.

\section{RESULTS}

\section{Attention Switching Task}

One participant in the 60-69 years group was excluded from the attention switching task analyses due to achieving chance level visual search accuracy in several conditions, including the Distractor Only pop-out search condition (mean $=0.40$ ), the Target Mid pop-out search condition (mean $=0.53$ ), and the Target Mid serial search condition (mean $=0.57$ ). The participant's low proportion of correct visual search responses indicates that they may not have understood the task. One participant from the $70+$ years group was excluded from RT analyses due to poor visual search and RSVP target identification, resulting in less than one third of serial search trials remaining in the Target 1st condition. Nineteen participants remained in the 70+ years group in the RT analysis and there were 20 participants in 60-69 years group in the remaining analysis.

\section{RSVP Accuracy}

Both the ability to detect targets in the RSVP stream and the proportion of correctly identified targets, where the participant correctly reported the target digit, were examined. Poor target detection would suggest that participants have a deficit in temporal selective attention. Group differences in target identification and not target detection may indicate a deficit in consolidation or recall of the target. Thus, distinguishing between correctly detected and identified targets could reveal specific age-related deficits in different cognitive processes.

\section{Target Detection}

A $2 \times 5$ (RSVP condition $\times$ age group) ANOVA was conducted on measures of $\mathrm{D}^{\prime}$, an index of target detection sensitivity. $\mathrm{D}^{\prime}$ provides a measure of detection sensitivity while controlling for false positive response rates, which has been shown to be inflated in older participants (Kenemans et al., 1995; Bolton and 
Staines, 2012). D' has previously been used as a measure of target sensitivity in work on visual attention in the ageing population (Parasuraman et al., 1989; Mouloua and Parasuraman, 1995; Berardi et al., 2001). D' for each RSVP condition are presented for each age group in Figure 2.

There were significant main effects of age $\left(F_{(4,95)}=9.04\right.$, $\left.p<0.001, \eta_{\mathrm{p}}^{2}=0.28\right)$ and RSVP condition $\left(F_{(4,95)}=43.55\right.$, $\left.p<0.001, \eta_{\mathrm{p}}^{2}=0.31\right)$ on $\mathrm{D}^{\prime}$. There was no significant interaction between age and RSVP condition $(p>0.10)$.

\section{Main effect of age}

Post hoc comparisons revealed that the main effect of age on detection sensitivity resulted from greater detection sensitivity in the 21-30 years group in comparison to the 50-59 $(p=0.036)$, $60-69(p<0.001)$ and $70+$ years $(p<0.001)$ groups. The 40-49 years group displayed a significantly higher detection sensitivity than the 60-69 $(p=0.005)$ and $70+$ years groups $(p=0.001)$. There were no other significant group differences in detection sensitivity $(p>0.10)$.

No further analysis was carried out on $\mathrm{D}^{\prime}$. Age differences in target detection suggest that difficulties derive from declines in selective attention that will similarly affect RSVP target identification, as is evident in Figure 2. Instead, target identifications were examined in more depth.

\section{Target Identification}

Figure 2 illustrates a decrease in target identification with increased age. A $2 \times 5$ (RSVP condition $\times$ age group) mixed ANOVA was conducted on the proportion of correctly identified RSVP targets.
There was a significant main effect of age $\left(F_{(4,95)}=9.06\right.$, $\left.p<0.001, \eta_{\mathrm{p}}^{2}=0.28\right)$ and RSVP condition $\left(F_{(1,95)}=43.40\right.$, $\left.p<0.001, \eta_{\mathrm{p}}^{2}=0.31\right)$ on RSVP target identification, as well as a significant age $\times$ RSVP condition interaction $\left(F_{(4,95)}=3.15\right.$, $p=0.018, \eta_{\mathrm{p}}^{2}=0.12$ ).

\section{Main effect of age}

It was hypothesized that the $70+$ years age group would identify fewer targets in comparison to younger groups but that there would be no difference in the proportion of targets identified in the 60-69 years age group. Post hoc comparisons showed that the main effect of age resulted from the 21-30 years group identifying significantly more RSVP targets than the 50-59 ( $p=0.017)$, $60-69(p=0.001)$ and $70+$ years $(p<0.001)$ groups. The $40-49$ years group identified significantly more targets than the $70+$ years group $(p=0.002)$. The higher target identification in the 40-49 years group in comparison to the 60-69 years group did not reach significance $(p=0.078)$. There were no other significant group differences in target identification $(p>0.10)$.

\section{Main Effect of RSVP Condition}

The main effect of RSVP condition resulted from participants identifying more targets in the Target 1st than the Target Mid condition.

\section{Interaction between age and RSVP Conditions}

To further explore the interaction between age group and RSVP condition on target identification independent $t$-tests were implemented to compare age groups on RSVP target identification on each RSVP condition separately.

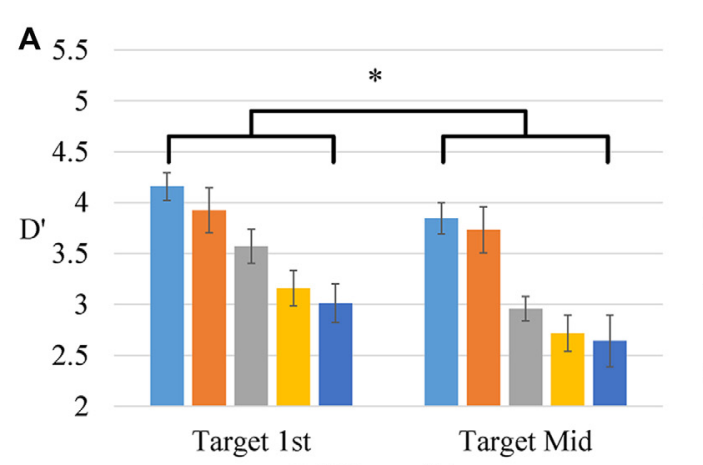

RSVP condition

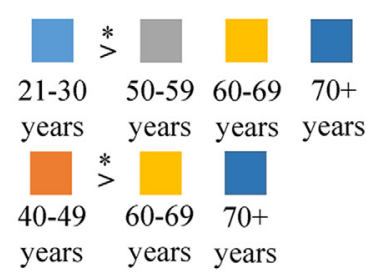

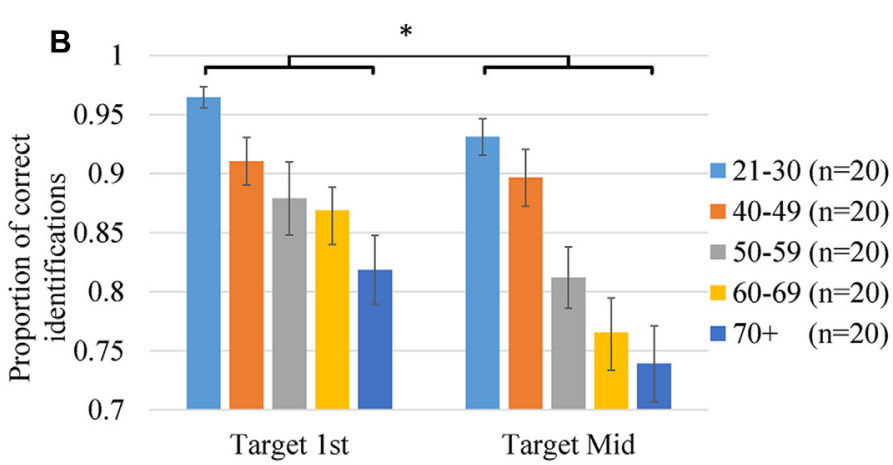

RSVP Condition

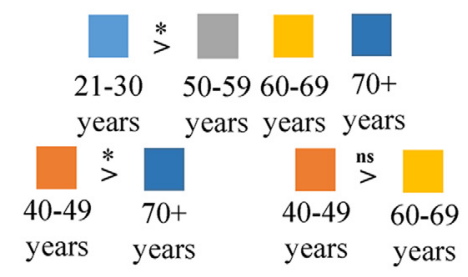

FIGURE 2 | RSVP Accuracy. An index of RSVP target detection sensitivity, D' (A) and the proportion of correctly identified RSVP targets (B) in each RSVP condition for each age group. The asterisk above each graph represents significant differences between RSVP conditions, collapsed across age groups. The color coded boxes below each graph illustrate significant age group differences collapsed across RSVP conditions. Vertical bars represent the standard error of the mean. 
In the Target 1 st condition, the 21-30 years group identified significantly more targets than the 40-49 $\left(_{(26.24)}=2.46\right.$, $p=0.021), 50-59\left(t_{(22.14)}=2.65, p=0.015\right), 60-69\left(t_{(26.77)}=4.49\right.$, $p<0.001)$ and $70+\left(t_{(22.54)}=4.79, p<0.001\right)$ years groups, and the $40-49$ years group identified more targets than the $70+$ years group $\left(t_{(38)}=2.60, p=0.013\right)$.

In the Target Mid condition, the 21-30 years group identified significantly more targets than the 50-59 $\left(t_{(38)}=3.93, p<0.001\right)$, 60-69 $\left(t_{(28.76)}=5.00, p<0.001\right)$ and $70+\left(t_{(27.31)}=5.39\right.$, $p<0.001)$ years groups, the 40-49 years group identified significantly more targets than the $50-59\left(t_{(38)}=2.38, p=0.022\right)$, $60-69\left(t_{(38)}=3.45, p=0.001\right)$ and $70+\left(t_{(38)}=3.92, p<0.001\right)$ years groups, and there was a non-significant trend for the 50-59 years group to identify more targets than the $70+$ years group $\left(t_{(38)}=1.77, p=0.086\right)$.

\section{Visual Search}

All groups correctly identified over $94 \%$ of visual search targets in all six conditions. Thus, no further analysis was carried out on visual search accuracy.

A $2 \times 3 \times 5$ (visual search condition $\times$ RSVP condition $\times$ age group) mixed ANOVA was conducted with participants' median RTs. Mauchly's Test of Sphericity was significant for RSVP condition $\left(\chi_{(2)}^{2}=8.56, p=0.014\right)$ indicating that the assumption of sphericity has been violated. Greenhouse-Geisser corrected statistics were therefore reported. Mean visual search RTs for each age group for serial and pop-out searches are presented in Figure 3.

Significant main effects of age $\left(F_{(4,94)}=13.39, p<0.001\right.$, $\left.\eta_{\mathrm{p}}^{2}=0.36\right)$, visual search condition $\left(F_{(1,94)}=335.17, p<0.001\right.$, $\left.\eta_{\mathrm{p}}^{2}=0.78\right)$, and RSVP condition $\left(F_{(1.84,172.80)}=133.57\right.$, $\left.p<0.001, \eta_{\mathrm{p}}^{2}=0.59\right)$ on visual search RTs were revealed, in addition to a significant age $\times$ visual search condition interaction $\left(F_{(4,94)}=4.98, p=0.001, \eta_{\mathrm{p}}^{2}=0.18\right)$, a significant age $\times$ RSVP condition interaction $\left(F_{(7.35,172.80)}=2.72, p=0.009, \eta_{\mathrm{p}}^{2}=0.10\right)$, and a significant visual search condition $\times$ RSVP condition interaction $\left(F_{(1.99,187.19)}=5.37, p=0.005, \eta_{\mathrm{p}}^{2}=0.05\right)$. There was no significant age $\times$ visual search condition $\times$ RSVP condition interaction $(p>0.10)$.

\section{Main effects of age}

Based on widely acknowledged age-related slowing of RTs, RTs were expected to increase with increased age (Salthouse, 1985, 2000; Foster et al., 1995). Post hoc comparisons illustrated that the 21-30 years group was significantly faster than the 50-59 $(p=0.024), 60-69(p<0.001)$ and $70+(p<0.001)$ years groups, but not the $40-49$ years group $(p>0.10)$. The $70+$ years group was slower than both the $40-49(p=0.001)$ and $50-59$ years groups $(p=0.004)$. There were no other significant group differences in RT $(p>0.10)$.

\section{Main effects of visual search condition}

Participants were significantly faster on the pop-out in comparison to serial visual search.

\section{Main effects of RSVP condition}

We hypothesized that RTs would be faster on the no-switch (Target 1st) condition, when participants no longer need to attend to the RSVP stream after identifying the target digit, in comparison to when they are required to attend to the RSVP stream to the end of the stream in the two switch conditions (Target Mid/Distractor only). The main effect of RSVP condition on visual search RTs resulted from significantly faster RTs on the Target 1st condition in comparison to both the Distractor Only $(p<0.001)$ and Target Mid $(p<0.001)$ conditions. There was no significant difference between the Distractor Only and Target Mid conditions $(p>0.10)$.

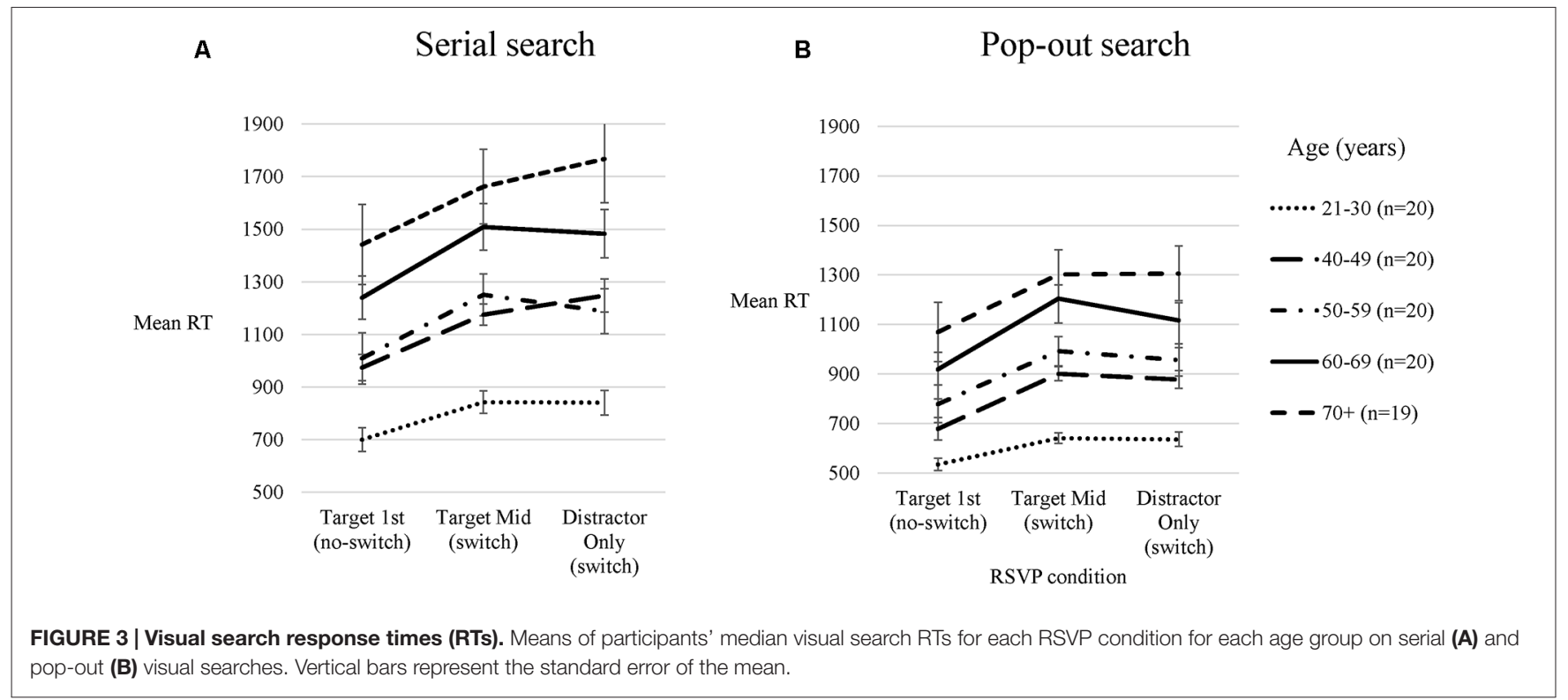




\section{Interaction between age and visual search conditions}

It is well established that older participants display deficits in serial but not in pop-out visual searches (Plude and Doussardroosevelt, 1989; Foster et al., 1995; Humphrey and Kramer, 1997; Bennett et al., 2012; Li et al., 2013). It was hypothesized that the increase in RTs on serial in comparison to pop-out search would be greater in older than younger groups. In support of this hypothesis, there was a significant age $\times$ visual search condition interaction. To investigate this hypothesis further, the percentage increase in RTs from the pop-out to serial search, collapsed across RSVP conditions, was calculated and entered into independent $t$-tests to compare groups. To collapse visual search RTs across RSVP conditions separately for serial and pop-out search RTs, each participant's median RTs was averaged across RSVP conditions separately for the pop-out search RTs and the serial search RTs. The mean percentage increase in RTs from pop-out to serial search is presented in Table 3.

Independent $t$-tests revealed that there was a significantly larger difference between serial and pop-out search RTs in the $40-49$ years than the $50-59\left(t_{(38)}=2.89, p=0.007\right)$. The larger difference between pop-out and serial search RTs in the $40-49$ years group in comparison to the 21-30 $\left(t_{(38)}=-1.85\right.$, $p=0.072), 60-69\left(t_{(38)}=1.80, p=0.080\right)$ and $70+(p>0.10)$ years groups did not reach significance. There were no further significant group differences in the percentage increase in RT from pop-out to serial search $(p>0.10)$.

\section{Interaction between age and RSVP conditions}

It was hypothesized that there would be greater difficulties in switching between the temporal and spatial attention tasks with increased age. To investigate the hypothesis that switchcosts would be greater with increased age, the interaction between age and RSVP condition was further explored. Each participant's percentage increases in RTs from the no-switch (Target 1st) condition to each of the switch conditions (Target Mid/Distractor Only) were calculated as measures of switchcosts. Collapsing visual search conditions to calculate switchcosts lead to finding no significant age group differences in switch-costs $(p>0.10)$. Thus, although there was no three-way interaction between age, visual search condition and RSVP condition $(p>0.10)$, switch-costs were calculated separately for serial and pop-out search RTs to gain a detailed understanding of the interaction between age and RSVP conditions. The resulting measures of switch-costs were entered into independent $t$-tests to compare groups. It is important to note that $t$-tests were exploratory, however, remain in the scope of current hypotheses. The means and standard deviations of each group's switch- costs on serial search and pop-out search RTs are presented in Table 4.

The percentage increase in pop-out search RTs from the Target 1 st to Target Mid condition were significantly greater for both the $40-49\left(t_{(38)}=-2.39, p=0.022\right)$ and $60-69$ years groups in comparison to the 21-30 years group $\left(t_{(38)}=-2.28\right.$, $p=0.028)$. The greater switch-costs in the $50-59$ years group in comparison to the 21-30 years groups did not reach significance $\left(t_{(38)}=-1.73, p=0.091\right)$. There were no significant differences in switch-costs between any other age groups for either visual search condition $(p>0.10)$.

\section{Interaction between visual search conditions and RSVP conditions}

No further analysis was carried out on the interaction between RSVP condition and visual search condition, as it is unrelated to the current hypotheses.

\section{Cognitive Function}

The cognitive mechanisms that underpin switching between modalities of attention were explored. Mean scores on UFOV processing speed, divided attention and selective attention, and the RNG index (inhibition) and R (updating) scores (Miyake et al., 2000) can be found in Table 5.

\section{The Relationship between Switch-Costs and Cognition}

To identify cognitive functions that may affect switching ability, the relationships between switch-costs and cognitive measures were examined separately for each age group. Relationships were examined only for switch-costs in the target-switch pop-out search condition, as it was in this condition only that age group differences were found. Shapiro Wilks test of normality demonstrated that the distribution of scores from all cognitive measures except the RNG index violated the assumption of normality for one or more age groups $(p<0.05)$. Spearman's rho correlation coefficients are therefore reported, which can be found in Table 6. Correlation strengths are interpreted based on Cohen $(1992,1988)$. It should be noted that correlations were exploratory and corrections for multiple comparisons were not conducted.

\section{UFOV Processing Speed}

There was a significant negative moderate correlation between switch-costs and UFOV processing speed in the 60-69 years group $(p=0.033)$. Those with greater switch-costs displayed faster processing speeds. The correlation between switch-costs

TABLE 3 | Means and standard deviations of the percentage difference from Pop-out to Serial search RTs for each group.

\begin{tabular}{|c|c|c|c|c|c|c|}
\hline & & \multicolumn{5}{|c|}{ Age Group (years) } \\
\hline & & $\begin{array}{c}21-30 \\
(n=20)\end{array}$ & $\begin{array}{c}40-49 \\
(n=20)\end{array}$ & $\begin{array}{c}50-59 \\
(n=19)\end{array}$ & $\begin{array}{c}60-69 \\
(n=20)\end{array}$ & $\begin{array}{c}70+ \\
(n=20)\end{array}$ \\
\hline Percentage & Mean & 21.68 & 27.21 & 19.21 & 22.65 & 23.17 \\
\hline Difference & SD & 11.01 & 7.61 & 9.95 & 8.41 & 8.58 \\
\hline
\end{tabular}

Percentage difference indicates the percentage increase in serial search RTs in comparison to pop-out search RTs. 


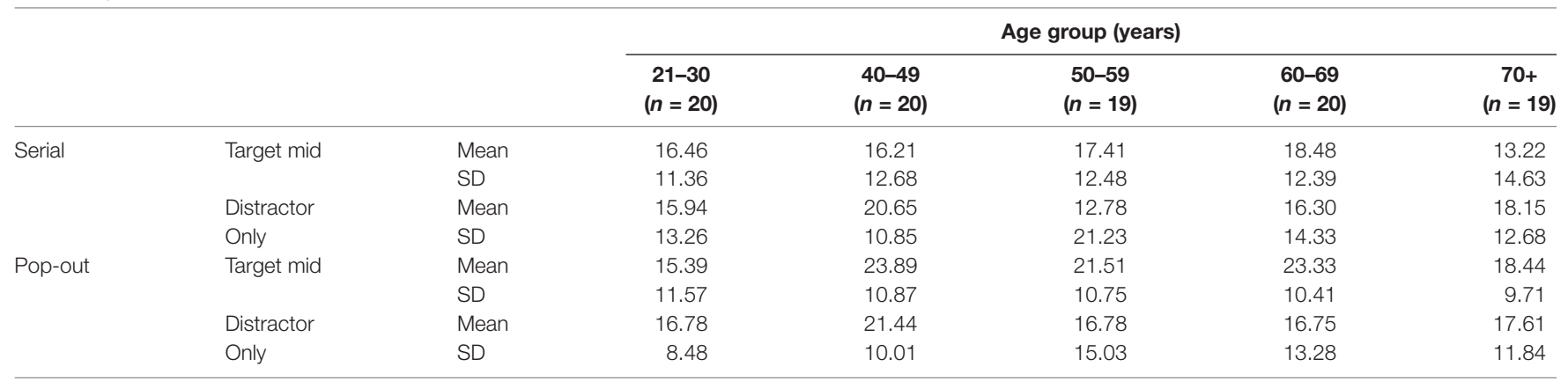

Switch-costs were calculated as the percentage increase in RT from the no-switch (Target 1st) condition to each of the switch conditions (Target Mid/Distractor Only) separately and separately for each visual search condition.

and processing speed did not reach significance in the 50-59 years group $(p=0.083)$. There were no other significant correlations between switch-costs and processing speed $(p>0.10)$.

\section{UFOV Divided Attention}

In the 50-59 years group there was a significant negative moderate correlation between switch-costs and UFOV divided attention $(p=0.027)$. Those with greater switch-costs performed better on the UFOV divided attention task (i.e., had faster processing thresholds). There were no other significant correlations between UFOV divided attention and switchcosts in any other age group $(p>0.10)$.

\section{UFOV Selective Attention}

There was a significant negative strong correlation between switch-costs and UFOV selective attention in the 50-59 years group $(p<0.001)$ and a non-significant negative moderate correlation between switch-costs and selective attention in the 60-69 years groups $(p=0.061)$. Participants with greater switchcosts had faster processing thresholds in the selective attention task. There were no other significant correlations between UFOV selective attention and switch-costs in any other age group $(p>0.10)$.

The direction of the relationship between switch-costs and performance on processing speed, divided attention and selective attention UFOV tasks was unexpected, as poor performance on the UFOV tasks was related to smaller switch-costs. These findings may be explained by a significant positive correlation between visual search RTs in the Target 1st condition and UFOV on processing speed ( $r=0.445, p<0.001, n=99)$, divided attention $(r=0.592, p<0.001, n=99)$ and selective attention $(r=0.577, p<0.001, n=99)$. Those who perform poorly on the UFOV tasks have slower visual search RTs on the Target 1st condition. Slow RTs on the Target 1st condition result in smaller switch-costs, as the difference between switch and no-switch conditions becomes smaller.

\section{RNG Task}

There were no significant correlations between switch-costs and RNG measures R or RNG in any age group $(p>0.10)$.

\section{DISCUSSION}

The aim of the current study was to investigate whether there is an age-related decline in the ability to switch between temporal and spatial attention and to explore the cognitive mechanisms that might underpin these changes. Identifying age-related cognitive changes that affect driving behavior is an important first step in working towards developing a cognitive intervention to improve driving performance and prolong the length of time that people are able to continue to drive.

There were decreases in both RSVP target detection sensitivity and target identification with increased age. Deficits in target identification but not target detection would suggest that group differences are related to memory and not temporal attention. Results therefore indicate that older participants' impaired performance derives from temporal attention mechanisms and results are not due to memory difficulties. It was hypothesized that there would be age-related difficulties in target identification in the $70+$ years age group but not the 60-69 years age group. On the contrary, the 21-30 years group identified more targets than all other age groups in both the Target 1st condition and the Target Mid condition. Age group differences were more extensive in the Target Mid condition in comparison to the Target 1st condition, and significantly fewer targets were identified in the Target Mid condition in comparison to the Target 1st condition overall. Poorer target identification in the Target Mid condition likely results from the presence of distractor stimuli both forward and backward masking Target Mid targets, whereas Target 1st targets were only backward masked. It is likely that the effect of distractors masking the target was further exacerbated by older adults' inhibitory deficits (Adamo et al., 2003; Maciokas and Crognale, 2003).

Consistent with previous research and with expectations, RTs were slower on serial than pop-out searches (Wolfe, 1998). These findings are due to attention being immediately drawn to the distinct target in pop-out searches, in contrast to when needing to complete a serial search (Treisman, 1985). Consistent with age-related slowing of RTs (Salthouse, 2000; Verhaeghen and Cerella, 2002), and supporting current hypotheses, there was an age-related increase in visual search RTs. 
TABLE 5 | Means and standard deviations for cognitive measures.

\begin{tabular}{|c|c|c|c|c|c|c|}
\hline & & \multicolumn{5}{|c|}{ Age group (years) } \\
\hline & & $\begin{array}{c}21-30 \\
(n=20)\end{array}$ & $\begin{array}{c}40-49 \\
(n=20)\end{array}$ & $\begin{array}{c}50-59 \\
(n=20)\end{array}$ & $\begin{array}{c}60-69 \\
(n=21)\end{array}$ & $\begin{array}{c}70+ \\
(n=21)\end{array}$ \\
\hline \multirow[t]{2}{*}{ Updating (R) } & Mean & 1.41 & 1.45 & 1.06 & 1.22 & 1.08 \\
\hline & SD & 1.78 & 1.07 & 0.81 & 0.52 & 0.71 \\
\hline \multirow[t]{2}{*}{ Inhibition (RNG) } & Mean & 0.33 & 0.34 & 0.32 & 0.34 & 0.36 \\
\hline & SD & 0.03 & 0.50 & 0.05 & 0.03 & 0.04 \\
\hline \multirow[t]{2}{*}{ Processing speed } & Mean & 16.72 & 16.70 & 21.54 & 25.44 & 22.54 \\
\hline & SD & 0.07 & 0.00 & 9.53 & 23.38 & 13.37 \\
\hline \multirow[t]{2}{*}{ Divided attention } & Mean & 24.22 & 39.05 & 58.20 & 49.26 & 79.04 \\
\hline & $\mathrm{SD}$ & 31.30 & 53.87 & 63.24 & 51.25 & 76.34 \\
\hline \multirow[t]{2}{*}{ Selective attention } & Mean & 54.39 & 101.36 & 127.08 & 151.50 & 186.06 \\
\hline & SD & 28.97 & 59.55 & 58.57 & 87.36 & 87.37 \\
\hline
\end{tabular}

One participant in the 70+ years group did not complete the UFOV, resulting in 20 participants in this group for the processing speed, divided attention and selective attention measures.

A greater increase in RTs from pop-out to serial search in the 40-49 years group in comparison to both younger and older groups was unexpected and contrasts with previous findings. Age-related deficits in serial but not pop-out search are well established (Plude and Doussardroosevelt, 1989; Foster et al., 1995; Humphrey and Kramer, 1997; Bennett et al., 2012; Li et al., 2013). The absence of greater differences between visual search conditions in the older groups may be due to ceiling effects, with slow RTs in both visual search conditions. In contrast, RTs on the pop-out search in the 40-49 years group remain fast and result in a larger percentage increase in RT from the pop-out to serial search.

Consistent with predictions, RTs were faster when switching from the RSVP task when the target was the first item in the stream in comparison to when the target was absent or in the middle of the stream. This can be attributed to larger switch-costs when attending to the RSVP stream to near the end of the stream than when able to disengage from the stream. These finding show that costs in switching from the temporal attentional task cause a delay to initiating a visual search.

Larger switch-costs in the $40-49$ and $60-69$ years groups in comparison to the youngest group for the Target Mid condition only partially supports the hypothesis that there would be increased switch-costs with age. Greater age-related switch-costs in only the 40-49 and 60-69 years age groups raises the question of why the 70+ years groups did not also display greater switchcosts in comparison to the youngest group and why the larger switch-costs in the 50-59 years group in comparison to the youngest group did not reach significance. RTs in the $40-49$ years group were not significantly slower than RTs in the youngest group. It may be that fast RTs in the Target 1st condition are inflating the switch-costs in the $40-49$ years group, as the percentage increase in RTs when they have to switch is greater. In contrast, switch-costs in the 50-59 years group are partially masked by their slow RTs in the Target 1st condition.

The question remains as to why greater switch-costs are seen in the 60-69 years and not in the 70+ years group. One explanation may be that the oldest group have developed efficient compensation strategies that are not yet present in the 60-69 years group. It may become necessary to adapt new strategies and recruit wider neural networks with older age due to increasingly impaired attentional and switching mechanisms combined with slowed RTs, whereas faster RTs in younger participants are sufficient to compensate for impaired switching. The recruitment of broader neural circuits with increased age is widely supported (Toepper et al., 2014), including in frontoparietal regions during attentional tasks (Madden et al., 2007), although it is unclear whether wider activation is due to compensation (Madden, 2007; Madden et al., 2007) or increased noise due to deficits in inhibitory mechanisms (Fabiani et al., 2006; Gazzaley et al., 2008). This raises the possibility that greater switch-costs were not seen in the $70+$ years group due to increased variability in RTs masking switch-costs. Increased variability masking greater switch-costs in the $70+$ years age group is supported by the increased variability observed with age in the current data.

TABLE 6 | Spearman's rho correlation coefficients, correlating cognitive measures with switch-costs.

\begin{tabular}{|c|c|c|c|c|c|}
\hline & \multicolumn{5}{|c|}{ Age group (years) } \\
\hline & $\begin{array}{c}20-39 \\
(n=20)\end{array}$ & $\begin{array}{c}40-49 \\
(n=20)\end{array}$ & $\begin{array}{c}50-59 \\
(n=20)\end{array}$ & $\begin{array}{c}60-69 \\
(n=20)\end{array}$ & $\begin{array}{c}70+ \\
(n=19)\end{array}$ \\
\hline RNG & -0.16 & -0.32 & 0.29 & 0.21 & -0.14 \\
\hline Processing speed & -0.06 & - & -0.40 & $-0.49^{*}$ & 0.14 \\
\hline Divided attention & 0.22 & 0.04 & $-0.50^{*}$ & 0.07 & -0.12 \\
\hline
\end{tabular}

${ }^{*} p<0.05,{ }^{* * *} p<0.001$; Updating (R), inhibition (RNG). 
A common limitation in aging research is self-selection bias. Older volunteers tend to be healthy, highly educated people who seek to stay active in later life. Both a physically, socially and cognitively active lifestyle, and higher levels of education and occupation have been shown to be protective factors against cognitive decline (Anstey and Christensen, 2000; Fratiglioni et al., 2004; López et al., 2014) and aspects of lifestyle such as level of education, video gaming habits and employment status have been shown to predict performance in visual attention tasks (Wilms and Nielsen, 2014). Thus, sample attributes may result in switch-costs in the 60-69 but not 70+ years, where there is less of a bias towards healthy, highly motivated people. However, the $70+$ years group did not display a significantly higher level of education and did not perform better on the ACE-3, which is a basic measure of cognitive function.

As a third alternative, the difference between switch and no-switch conditions may have been reduced in the 70+ years group due to participants taking longer to process the Target 1st target and/or taking longer to disengage attention from the RSVP stream in the no-switch condition due to difficulties in inhibiting distractor stimuli. In both scenarios, visual search RTs in the no-switch condition would be inflated, reducing the difference between switch and no-switch conditions. These explanations would be consistent with increased visual processing speeds with increased age (Ball et al., 2006; Rubin et al., 2007) and with evidence that suggests that temporal attention is impaired only in those over the age of 70 years and not in those aged 60-69 years (Lee and Hsieh, 2009; Shih, 2009), explaining why increases in switch-costs are seen in the 60-69 years group and not the $70+$ years group. This explanation would also account for the surprising findings of increased switch-costs with faster processing speed thresholds in the UFOV processing speed and selective attention tasks that were seen in both the 50-59 and 60-69 years groups. This relationship was in the opposite direction to expectations. To perform well on the UFOV selective attention task, one is required to inhibit irrelevant distractors across the screen to selectively attend to the target. Thus, inhibitory deficits seem to be resulting in both a smaller difference between no-switch and switch conditions, due to difficulties in disengaging from the RSVP stream, and longer processing speeds in the UFOV selective attention task. However, it is important to note that correlation analyses were exploratory and corrections for multiple comparisons were not conducted. Further research with larger sample sizes is needed to corroborate these findings.

However, switch-costs did not correlate with the RNG index, which is a measure of inhibition. This may be because inhibitory mechanisms implemented in the RNG task to inhibit repetition and number sequences are separate from those involved in inhibiting visual distractors. Excitatory-inhibitory competition in the visual cortex is involved in selectively attending visual information (Beck and Kastner, 2009; Reynolds et al., 1999), whereas inhibition during the RNG task is likely to involve the inhibition of response in working memory localized to the prefrontal cortex (Daniels et al., 2003). This conclusion is supported by Madden et al. (2007) who, in a serial visual search task, found that whereas young adults' performance was associated with occipital lobe activation, older adults' performance was more strongly related to frontal and parietal activity. These findings are consistent with a specific decline in serial search performance with age caused by deficits in excitatory-inhibitory mechanisms during visual processing.

Age differences in switch-costs in the Target Mid condition and not in the Distractor Only condition are likely due to the requirement to consolidate the RSVP target. It could be that increased switch-costs in this condition are due to slow processing speeds resulting in participants taking longer to process the target, which delays the switch to allocate attention spatially. On the contrary, fast processing speeds were related to increased switch-costs. Current findings therefore suggest that deficits in switching between temporal and spatial attention were not due to general slowing.

The current results support Lee and Hsieh (2009) findings of an age-related increase in difficulties in switching from attending to an RSVP stream to identify a target, to allocating attention in space to identify and point to a masked peripheral target. However, Lee and Hsieh (2009) aim was to investigate the attentional blink in older adults, and thus does not distinguish between impaired task performance resulting from an increased attentional blink, or due to deficits in switching between temporal and spatial attention. The inclusion of the Distractor Only condition in the current task enabled the investigation of whether age-related deficits in switching were due to increases in the time taken to switch between attentional mechanisms or an increased attentional blink. If there was a deficit in switching between attentional mechanisms, then older adults should be impaired in switching in both the Distractor Only and Target Mid conditions when compared with younger adults. Conversely, the current findings of increased switchcosts in the Target Mid condition only indicate that higher switch-costs may result from an increased attentional blink after processing the RSVP target. However, Figure 3 could also indicate that increased variability in the Distractor Only condition may have prevented group differences from emerging (e.g., $70+$ group). Despite attempts to minimize variability in RTs by using an initial space-bar response, high variability masking differences in statistical power is corroborated by generally increased variability in RTs with increased age in the current dataset. The absence of a significant difference in RTs between the Distractor Only condition and the Target Mid condition further supports that group differences in switch-costs in the Distractor Only condition were not seen due to variability in RTs masking differences in statistical power.

Further work is required to explore how age-related declines in switching translate to driving behavior. It may be that difficulties in switching between temporal and spatial attention cause difficulties in switching from attending to traffic on the road ahead to attend to road signs and other surrounding objects. The current authors are presently 
exploring how attention switching predicts simulated driving performance. Previous work has shown that age-related difficulties in selective attention affect driving ability in some older drivers but not others (Vaucher et al., 2014). It may be that similarly, difficulties in switching between modalities of attention negatively affect the performance of some drivers but not others. If difficulties in switching are found to affect driving performance, then it will be important to develop an intervention to improve switching between modalities of attention to help improve driver performance and safety. Long-term, this will prolong the time that older drivers can continue to drive and help to preserve their independence.

\section{Limitations}

In contrast to previous visual search paradigms (Humphrey and Kramer, 1997; Li et al., 2013), participants were required to make an initial space-bar response to indicate that they had identified the target and then report which letter they had seen. A limitation of this approach is that participants may modify their decision after they have made a response with the aid of visual memory. It is not known whether the ability to adopt this strategy is greater in young adults than older adults. However, the opportunity to implement this strategy was present in both switch and no-switch trials and therefore should not have affected our main findings.

A further limitation of the current paradigm is that we did not explore how switching affects the ongoing visual search processes, as the number of distractor stimuli in the visual search display was not manipulated. In the current paradigm we were interested in the efficiency of switching to initiate a search. Further research is required to investigate how switching influences ongoing search processes. It may be that switching has a large effect on search speed at the beginning of a search but the effect on search speed plateaus with increasing numbers of distractors, as time since the switch increases. Furthermore, it may be that this switch-cost is not specific to switching between types of attention, but would also affect performance in other cognitive functions. Although it is switching between temporal and spatial attention that is important to driving, where declines in efficiency may have a negative impact on a person's life, this difficulty may generalize to other tasks. This is an important question to ask when developing an intervention.

The 40-49 and 50-59 years age groups were intended as middle-age comparison groups for the two oldest age groups and the 21-30 years group was intended as a comparison group for all other age groups. The finding of higher switch-costs in the 40-49 years group was unexpected, particularly as no differences in RTs were found between the 21-30 and 40-49 years groups. Future research would benefit from also including a 30-39 years age group in order to obtain a view of how the ability to switch between attentional mechanisms changes throughout the adult lifespan.

It is well established that working memory capacity declines with healthy aging, including both verbal (Hultsch et al., 1992; Zacks et al., 2000) and visual (Faubert, 2002;
Brockmole and Logie, 2013) short term memory. A limitation of the current study is that no measure of verbal or visual working memory capacity was taken to look at the influence of memory on switching. However, the strain on working memory is very low, as the participant is only required to hold a single item in memory (i.e., the RSVP target digit). It is therefore unlikely that difficulties in working memory would affect switching performance. Furthermore, Akyürek and Hommel (2005) found that working memory load did not interact with the duration of the attentional blink. Additionally, working memory load remains constant across both the no-switch and Target Mid switch conditions and so memory deficits should not have influenced our main findings.

Although age-related declines in working memory capacity are unlikely to have affected switching performance in the current task, it is possible that declines in executive function affected switching performance. In relation to Baddeley's (1992) working memory model, the current task would require the top-down control of attention from the central executive. It was therefore expected that measures of executive function would predict switching performance. However, measures of executive function obtained from the RNG did not correlate with task performance. It was found that there were no age group differences in RNG measures, despite age-related declines in executive function being widely acknowledged in the literature (Cepeda et al., 2001; Gamboz et al., 2009; Gold et al., 2010). It may be that RNG performance is too susceptible to interference from the use of alternative strategies, such as visualization techniques. Further research is needed to explore the relationship between executive function and switching between temporal and spatial attention to come to more sound conclusions.

A further limitation of the methodology is that eye tracking data were not recorded and participants' actual fixation was not controlled for to ensure that participants were focusing on the visual search fixation cross. Participants' failure to focus on the fixation cross could result in error in the measurements of RTs to complete the visual search. However, participants were instructed to keep their eyes fixed on the fixation cross, a protocol that is commonly used across cognitive paradigms (Humphrey and Kramer, 1997; Watson and Maylor, 2002; Li et al., 2013). Furthermore, participants' attention to the RSVP stream before the onset of the visual search display ensured that participants were focusing on the center of the screen at the beginning of each trial. Trials in which participants failed to correctly identify the target digit in the RSVP stream were excluded from RT analyses, which ensured that only trials in which participants were attending to the task were included in analyses.

Gender differences in the decline of certain attentional mechanisms have previously been found (Conlon and Herkes, 2008). The current study did not look at gender differences in age-related changes in switching ability as it was beyond the scope of the study. Future work could investigate whether there are any gender differences in the ability to switch between temporal and spatial attention in older age. 


\section{Conclusions and Future Directions}

The hypothesis that there would be greater switch-costs in older than younger groups was partially supported, as people aged 40-49 and 60-69 years displayed greater switch-costs than those aged 20-29 years. There was also a non-significant trend for greater switch-costs in the 50-59 years group. Increased switchcosts in the 40-49 and 60-69 years groups but not the 70+ years groups was surprising. However, switching difficulties in the oldest group may have been masked by slow RTs on the Target 1st condition due to a failure to inhibit and disengage from the RSVP stream. This conclusion would explain the surprising findings of decreased switch-costs with slower selective attention processing speeds. Poor selective attention could mask switchcosts due to difficulties with inhibiting the remainder of the RSVP stream in the Target 1st condition resulting in slow RTs. Future studies investigating switching between temporal and spatial attention would benefit from including a condition that contains a target with no distractor stimuli.

Increased switch-costs in the Target Mid condition but not the Distractor Only condition indicates that increased switchcosts could result from either an increased attentional blink following RSVP target identification, which delays the allocation of attentional resources to the visual search, or increased variability in RTs in the Distractor Only condition.

The current authors are presently investigating whether age-related difficulties in switching affect driving performance.

\section{REFERENCES}

Adamo, M., Westerfield, M., Haist, F., and Townsend, J. (2003). "Cross-modal shifting attention in healthy aging: an fMRI study," in Poster Presented at Society of Neuroscience Abstract Viewer and Itinerary Planner, 3 (Toronto, ON: University of Toronto).

Akyürek, E. G., and Hommel, B. (2005). Short-term memory and the attentional blink: capacity versus content. Mem. Cognit. 33, 654-663. doi: 10.3758/bf03195332

Anstey, K., and Christensen, H. (2000). Education, activity, health, blood pressure and apolipoprotein $\mathrm{E}$ as predictors of cognitive change in old age: a review. Gerontology 46, 163-177. doi: 10.1159/000022153

Arai, A., and Arai, Y. (2015). Self-assessed driving behaviors associated with age among middle-aged and older adults in Japan. Arch. Gerontol. Geriatr. 60, 39-44. doi: 10.1016/j.archger.2014.10.017

Baddeley, A. (1992). Working memory. Science 255, 556-559. doi: 10.1126/science. 1736359

Ball, K. K., Beard, B. L., Roenker, D. L., Miller, R. L., and Griggs, D. S. (1988). Age and visual search: expanding the useful field of view. J. Opt. Soc. Am. A 5, 2210-2219. doi: 10.1364/josaa.5.002210

Ball, K. K., Owsley, C., Sloane, M. E., Roenker, D., and Bruni, J. R. (1993). Visual attention problems as a predictor of vehicle crashers in older drivers. Invest. Ophthalmol. Vis. Sci. 34, 3110-3123.

Ball, K. K., Roenker, D. L., Wadley, V. G., Edwards, J. D., Roth, D. L., McGwin, G., et al. (2006). Can high-risk older drivers be identified through performance-based measures in a department of motor vehicle setting? J. Am. Geriatr. Soc. 54, 77-84. doi: 10.1111/j.1532-5415.2005. 00568.x

Beck, D., and Kastner, S. (2009). Top-down and bottom-up mechanisms in biasing competition in the human brain. Vision Res. 49, 1154-1165. doi: 10.1016/j. visres.2008.07.012

Bennett, I. J., Motes, M. A., Rao, N. K., and Rypma, B. (2012). White matter tract integrity predicts visual search performance in young and older adults. Neurobiol. Aging 33, 433.e21-433.e31. doi: 10.1016/j.neurobiolaging.2011. 02.001
If difficulties in switching affect driving performance, then this cognitive process should be targeted in the development of interventions that aim to improve driver performance and safety. Long-term, this will prolong the time that older drivers can continue to drive.

\section{AUTHOR CONTRIBUTIONS}

EC contributed towards the design of the research, played a key role in data collection, data analysis and interpretation of the analysis, in addition to drafting and revising the written article and approving the final version to be published. $\mathrm{CH}$ contributed towards the conception and design of the work, to the data analysis and interpretation, in addition to contributing towards the critical revision of the article and approving the final version to be published. KK made a substantial contribution to the conception and design of the work, to the data analysis and interpretation, in addition to contributing towards the critical revision of the article and approving the final version to be published.

\section{ACKNOWLEDGMENTS}

This research was supported by funding from The Rees Jeffreys Road Fund and by the School of Life and Health Sciences at Aston University.

Berardi, A., Parasuraman, R., and Haxby, J. V. (2001). Overall vigilance and sustained attention decrements in healthy ageing. Exp. Aging Res. 27, 19-39. doi: 10.1080/03610730126014

Bolton, D. A. E., and Staines, W. R. (2012). Age-related loss in attention-based modulation of tactile stimuli at early stages of somatosensory processing. Neuropsychologia 50, 1502-1513. doi: 10.1016/j.neuropsychologia.2012.03.002

Brockmole, J. R., and Logie, R. H. (2013). Age-related change in visual working memory: a study of 55, 753 participants aged 8-75. Front. Psychol. 4:12. doi: 10.3389/fpsyg.2013.00012

Budescu, D. V. (1982). The power of the $F$ test in normal populations with heterogeneous variances. Educ. Psychol. Meas. 42, 409-416. doi: $10.1177 / 001316448204200202$

Budescu, D. V., and Appelbaum, M. I. (1981). Variance stabilizing transformations and the power of the F test. J. Educ. Behav. Stat. 6, 55-74. doi: 10.3102/10769986006001055

Bundesen, C., Habekost, T., and Kyllingsbæk, S. (2005). A neural theory of visual attention: bridging cognition and neurophysiology. Psychol. Rev. 112, 291-328. doi: 10.1037/0033-295X.112.2.291

Cepeda, N. J., Kramer, A. F., and Gonzalez de Sather, J. C. M. (2001). Changes in executive control across the life span: examination of task-switching performance. Dev. Psychol. 35, 715-730. doi: 10.1037/0012-1649.37.5.715

Cohen, J. (1988). Statistical Power Analysis for the Behavioural Sciences. 2nd Edn. New York, NY: Academic Press.

Cohen, J. (1992). A power primer. Psychol. Bull. 112, 155-159. doi: 10.1037/00332909.112.1.155

Conlon, E., and Herkes, K. (2008). Spatial and temporal processing in healthy aging: implications for perceptions of driving skills. Neuropsychol. Dev. Cogn. B Aging Neuropsychol. Cogn. 15, 446-470. doi: 10.1080/138255807018 78008

Coull, J. T., and Nobre, A. C. (1998). Where and when to pay attention: the neural systems for directing attention to spatial locations and to time intervals as revealed by both PET and fMRI. J. Neurosci. 18, 7426-7435.

Cuenen, A., Jongen, E. M. M., Brijs, T., Brijs, K., Lutin, M., Van Vlierden, K., et al. (2015). Does attention capacity moderate the effect of driver distraction in older drivers? Accid. Anal. Prev. 77, 12-20. doi: 10.1016/j.aap.2015.01.011 
Daniels, C., Witt, K., Wolff, S., Jansen, O., and Deuschl, G. (2003). Rate dependency of the human cortical network subserving executive functions during generation of random number series-a functional magnetic resonance imaging study. Neurosci. Lett. 345, 25-28. doi: 10.1016/s0304-3940(03)00496-8

Desimone, R. (1998). Visual attention mediated by biased competition in extrastriate visual cortex. Philos. Trans. R. Soc. Lond. B Biol. Sci. 354, 1245-1255. doi: 10.1098/rstb.1998.0280

Edwards, J. D., Delahunt, P. B., and Mahncke, H. W. (2009). Cognitive speed of processing training delays driving cessation. J. Gerontol. A Biol. Sci. Med. Sci. 64, 1-6. doi: 10.1093/gerona/glp131

Evans, F. J. (1978). Monitoring attention deployment by random number generation: an index to measure subjective randomness. Bull. Psychon. Soc. 12, 35-38. doi: 10.3758/bf03329617

Fabiani, M., Low, K. A., Wee, E., Sable, J. J., and Gratton, G. (2006). Reduced suppression or labile memory? Mechanisms of inefficient filtering of irrelevant information in older adults. J. Cogn. Neurosci. 18, 637-650. doi: 10.1162/jocn. 2006.18.4.637

Faubert, J. (2002). Visual perception and aging. Can J. Exp. Psychol. 56, 164-176.

Foster, J. K., Behrmann, M., and Stuss, D. T. (1995). Aging and visual search: generalized cognitive slowing or selective deficit in attention? Aging Neuropsychol. Cogn. 2, 279-299. doi: 10.1080/138255895082 56604

Fratiglioni, L., Paillard-Borg, S., and Winblad, B. (2004). An active and socially integrated lifestyle in late life might protect against dementia. Lancet Neurol. 3 , 343-353. doi: 10.1016/s1474-4422(04)00767-7

Fu, S. M., Caggiano, D. M., Greenwood, P. M., and Parasuraman, R. (2005). Eventrelated potentials reveal dissociable mechanisms for orienting and focusing visuospatial attention. Cogn. Brain Res. 23, 341-353. doi: 10.1016/j.cogbrainres. 2004.11.014

Gamboz, N., Borella, E., and Brandimonte, M. A. (2009). The role of switching, inhibition and working memory in older adults' performance in the Wisconsin Card Sorting Test. Neuropsychol. Dev. Cogn. B Aging Neuropsychol. Cogn. 16, 260-284. doi: 10.1080/13825580802573045

Gazzaley, A., Clapp, W., Kelley, J., McEvoy, K., Knight, R. T., and D’Esposito, M. (2008). Age-related top-down suppression deficit in the early stages of cortical visual memory processing. Proc. Natl. Acad. Sci. U S A 105, 13122-13126. doi: 10.1073/pnas.0806074105

Gold, B. T., Powell, D. K., Xuan, L., Jicha, G. A., and Smith, C. D. (2010). Age-related slowing of task switching is associated with decreased integrity of frontoparietal white matter. Neurobiol. Aging 31, 512-522. doi: 10.1016/j. neurobiolaging.2008.04.005

Greenwood, P. M., and Parasuraman, R. (1994). Attentional disengagement deficit in non-demented elderly over 75 years of age. Aging Neuropsychol. Cogn. 1, 188-202. doi: 10.1080/13825589408256576

Gross, J., Schmitz, F., Schnitzler, I., Kessler, K., Shapiro, K., Hommel, B., et al. (2004). Modulation of long-range neural synchrony reflects temporal limitations of visual attention in humans. Proc. Natl. Acad. Sci. U S A 101, 13050-13055. doi: 10.1073/pnas.0404944101

Guo, A. W., Brake, J. F., Edwards, S. J., Blythe, P. T., and Fairchild, R. G. (2010). The application of in-vehicle systems for elderly drivers. Eur. Transp. Res. Rev. 2, 165-174. doi: 10.1007/s12544-010-0037-y

Hakamies-Blomqvist, L. E. (1993). Fatal accidents of older drivers. Accid. Anal. Prev. 25, 19-27. doi: 10.1016/0001-4575(93)90093-c

Hale, S., Myerson, J., Smith, G. A., and Poon, L. W. (1988). Age, variability, and speed: between subjects diversity. Psychol. Aging 3, 407-410. doi: 10.1037/08827974.3.4.407

Hasher, L., and Zacks, R. T. (1988). Working memory, comprehension and aging: a review and a new view. Psychol. Learn. Motiv. 22, 193-225. doi: 10.1016/s00797421(08)60041-9

Hennessy, D. E. (1995). Vision Testing of Renewal Applicants: Crashes PredicteD When Compensation for Impairment is Inadequate. Sacramento, CA: Research and Development Section.

Hoffman, L., McDowd, J. M., Atchley, P., and Dubinsky, R. (2005). The role of visual attention in predicting driving impairment in older adults. Psychol. Aging 20, 610-622. doi: 10.1037/0882-7974.20.4.610

Horne, R., Evans, F. J., and Orne, M. T. (1982). Random number generation, psychopathology and therapeutic change. Arch. Gen. Psychiatry 39, 680-683. doi: 10.1001/archpsyc. 1982.04290060042008
Hultsch, D. F., Hertzog, C., Small, B. J., McDonald-Miszczak, L., and Dixon, R. A. (1992). Short-term longitudinal change in cognitive performance in later life. Psychol. Aging 7, 571-584. doi: 10.1037/0882-7974.7.4.571

Hultsch, D. F., MacDonald, S. W. S., and Dixon, R. A. (2002). Variability in reaction time performance of younger and older adults. J. Gerontol. B Psychol. Sci. Soc. Sci. 57, P101-P115. doi: 10.1093/geronb/57. 2.p101

Humphrey, D. G., and Kramer, A. F. (1997). Age differences in visual search for feature, conjunction and triple-conjunction targets. Psychol. Aging 12, 704-717. doi: 10.1037/0882-7974.12.4.704

Jefferies, L. N., Roggeveen, A. B., Enns, J. T., Bennett, P. J., Sekuler, A. B., and Di Lollo, V. (2015). On the time course of attentional focusing in older adults. Psychol. Res. 79, 28-41. doi: 10.1007/s00426-013-0528-2

Kenemans, J. L., Smulders, F. T. Y., and Kok, A. (1995). Selective processing of 2-dimensional visual-stimuli in young and old subjects: electrophysiological analysis. Psychophysiology 32, 108-120. doi: 10.1111/j.1469-8986.1995. tb03302.x

Lahar, C. J., Isaak, M. I., and McArther, A. D. (2001). Age differences in the magnitude of the attentional blink. Aging Neuropsychol. Cogn. 8, 149-159. doi: 10.1076/anec.8.2.149.842

Lee, T., and Hsieh, S. (2009). The limits of attention of visual perception and action in aging. Neuropsychol. Dev. Cogn. B Aging Neuropsychol. Cogn. 16, 311-329. doi: 10.1080/13825580902741351

Leversen, J. S. R., Hopkins, B., and Sigmundsson, H. (2013). Ageing and driving: examining the effects of visual processing demands. Transp. Res. Part F Traffic Psychol. Behav. 17, 1-4. doi: 10.1016/j.trf.2012.11.003

Li, L., Gratton, C., Fabiani, M., and Knight, R. T. (2013). Age-related frontoparietal changes during the control of bottom-up and top-down attention: an ERP study. Neurobiol. Aging 34, 477-488. doi: 10.1016/j.neurobiolaging.2012. 02.025

Lien, M., Gemperle, A., and Ruthruff, E. (2011). Aging and involuntary attention capture: electrophysiological evidence for preserved attentional control with advanced age. Psychol. Aging 26, 188-202. doi: 10.1037/a00 21073

López, M. E., Aurtenetxe, S., Pereda, E., Cuesta, P., Castellanos, N. P., Bruna, R., et al. (2014). Cognitive reserve is associated with the functional organization of the brain in healthy aging: a MEG study. Front. Aging Neurosci. 6:125. doi: 10.3389/fnagi.2014.00125

Luck, S. J., Girelli, M., McDermott, M. T., and Ford, M. A. (1997). Bridging the gap between the monkey neurophysiology and human perception: an ambiguity resolution theory of visual selective attention. Cogn. Psychol. 33, 64-87. doi: 10.1006/cogp.1997.0660

Lustig, C., Hasher, L., and Zacks, R. T. (2007). Inhibitory deficit theory: recent developments in a "new view". Inhibition in Cognition 17, 145-162. doi: 10.1037/11587-008

Maciokas, J. B., and Crognale, M. A. (2003). Cognitive and attentional changes with age: evidence from attentional blink deficits. Exp. Aging Res. 29, 137-153. doi: $10.1080 / 03610730303715$

Madden, D. J. (2007). Aging and visual attention. Curr. Dir. Psychol. Sci. 16, 70-74. doi: 10.1111/j.1467-8721.2007.00478.x

Madden, D. J., Spaniol, J., Whiting, W. L., Bucur, B., Provenzale, J. M., Cabeza, R., et al. (2007). Adult age differences in the functional neuroanatomy of visual attention: a combined fMRI and DTI study. Neurobiol. Aging 28, 459-476. doi: 10.1016/j.neurobiolaging.2006.01.005

Marottoli, R. A., Mendes de Leon, C. F., Glass, T. A., Williams, C. S., Cooney, L. M. Jr., Berkman, L. F., et al. (1997). Driving cessation and increased depressive symptoms: prospective evidence from the New Haven EPESE. J. Am. Geriatr. Soc. 45, 202-206. doi: 10.1111/j.1532-5415.1997.tb04508.x

McGwin, G. J. Jr., and Brown, D. B. (1999). Characteristics of traffic crashes among young, middle-aged and older drivers. Accid. Anal. Prev. 31, 181-198. doi: 10.1016/s0001-4575(98)00061-x

McLaughlin, P. M., and Murtha, S. J. E. (2010). The effects of age and exogenous support on visual search performance. Exp. Aging Res. 36, 325-345. doi: 10.1080/0361073X.2010.484752

Miyake, A., Friedman, N. P., Emerson, M. J., Witzki, A. H., Howerter, A., and Wager, T. D. (2000). The unity and diversity of executive functions and their contributions to complex "frontal lobe" tasks: a latent variable analysis. Cogn. Psychol. 41, 49-100. doi: 10.1006/cogp.1999.0734 
Morse, C. K. (1993). Does variability increase with age? An archival study of cognitive measures. Psychol. Aging 8, 156-164. doi: 10.1037/0882-7974.8.2.156

Mouloua, M., and Parasuraman, R. (1995). Aging and cognitive vigilance-effects of spatial uncertainty and event rate. Exp. Aging Res. 21, 17-32. doi: 10.1080/03610739508254265

Musselwhite, C. B. A., and Haddad, H. (2010). Exploring older drivers' perceptions of driving. Eur. J. Ageing 7, 181-188. doi: 10.1093/occmed/kqv041

Neider, M. B., and Kramer, A. F. (2011). Older adults capitalize on contextual information to guide search. Exp. Aging Res. 37, 539-571. doi: 10.1080/0361073X.2011.619864

Noone, P. (2015). Addenbrooke's cognitive examination-III. Occup. Med. (Lond) 65, 418-420. doi: 10.1007/s10433-010-0147-3

Norman, J. F., Ross, H. E., Hawkes, L. M., and Long, J. R. (2003). Aging and the perception of speed. Perception 32, 85-96. doi: 10.1068/p3478

Parasuraman, R., and Nestor, P. G. (1991). Attention and driving skills in aging and Alzheimer's disease. Hum. Factors 33, 539-557.

Parasuraman, R., Nestor, P. G., and Greenwood, P. (1989). Sustainedattention capacity in young and older adults. Psychol. Aging 4, 339-345. doi: 10.1037/0882-7974.4.3.339

Plude, D. J., and Doussardroosevelt, J. A. (1989). Aging, selective attention and feature integration. Psychol. Aging 4, 98-105. doi: 10.1037/0882-7974.4.1.98

Porter, G., Tales, A., Troscianko, T., Wilcock, G., Haworth, J., and Leonards, U. (2010). New insights into feature and conjunction search: I. Evidence from pupil size, eye movements and ageing. Cortex 46, 621-636. doi: 10.1016/j. cortex.2009.04.013

Quigley, C., Andeson, S. K., and Müller, M. M. (2012). Keeping focused: sustained spatial selective visual attention is maintained in healthy old age. Brain Res. 1469, 24-34. doi: 10.1016/j.brainres.2012.06.019

Ragland, D. R., Satariano, W. A., and MacLeod, K. E. (2005). Driving cessation and increased depressive symptoms. J. Gerontol. A Biol. Sci. Med. Sci. 60, 399-403. doi: 10.1093/gerona/60.3.399

Raymond, J. E., Shapiro, K. L., and Arnell, K. M. (1992). Temporary suppression of visual processing in an RSVP task: an attentional blink? J. Exp. Psychol. Hum. Percept. Perform. 18, 849-860. doi: 10.1037/0096-1523.18.3.849

Reynolds, J. H., Chelazzi, L., and Desimone, R. (1999). Competitive mechanisms subserve attention in macaque areas V2 and V4. J. Neurosci. 19, 1736-1753.

Richardson, E. D., and Marottoli, R. A. (2003). Visual attention and driving behaviors among community-living older persons. J. Gerontol. A Biol. Sci. Med. Sci. 58, M832-M836. doi: 10.1093/gerona/58.9.m832

Richardson, J. T. E., and Vecchi, T. (2002). A jigsaw-puzzle imagery task for assessing active visuospatial processes in old and young people. Behav. Res. Methods Instrum. Comput. 34, 69-82. doi: 10.3758/bf03195425

Rubin, G. S., Ng, E. S. W., Bandeen-Roche, K., Keyl, P. M., Freeman, E. E., and West, S. K. (2007). A prospective, population-based study of the role of visual impairment in motor vehicle crashes among older drivers: the SEE study. Invest. Ophthalmol. Vis. Sci. 48, 1483-1491. doi: 10.1167/iovs. 06-0474

Russell, C., Malhotra, P., Deidda, C., and Husain, M. (2013). Dynamic attentional modulation of vision across space and time after right hemisphere stroke and in ageing. Cortex 49, 1874-1883. doi: 10.1016/j.cortex.2012.10.005

Salthouse, T. A. (1985). A Theory of Cognitive Aging. Amsterdam: North-Holland.

Salthouse, T. A. (2000). Aging and measures of processing speed. Biol. Psychol. 54, 35-54. doi: 10.1016/s0301-0511(00)00052-1

Scalf, P. E., Torralbo, A., Tapia, E., and Beck, D. M. (2013). Competition explains limited attention and perceptual resources: implications for perceptual load and dilution theories. Front. Psychol. 4:243. doi: 10.3389/fpsyg.2013. 00243

Schiff, W., Oldak, R., and Shah, V. (1992). Aging persons' estimates of vehicular motion. Psychol. Aging 7, 518-525. doi: 10.1037/0882-7974.7.4.518

Scialfa, C. T., Guzy, L. T., Leibowitz, H. W., Garvey, P. M., and Tyrrell, R. A. (1991). Age differences in estimating vehicle velocity. Psychol. Aging 6, 60-66. doi: 10.1037/0882-7974.6.1.60

Shapiro, K., Hillstrom, A. P., and Husain, M. (2002). Control of visuotemporal attention by inferior parietal and superior temporal cortex. Curr. Biol. 12, 1320-1325. doi: 10.1016/s0960-9822(02)01040-0

Shih, S. I. (2009). Using the attention cascade model to prove cognitive ageing. Psychol. Aging 24, 550-562. doi: 10.1037/a0016724
Snedecor, G. W., and Cochran, W. G. (1967). Statistical Methods. Ames, IA: Iowa State University Press.

Snowden, R. J., and Kavanagh, E. (2006). Motion perception in the ageing visual system: minimum motion, motion coherence and speed discrimination thresholds. Perception 35, 9-24. doi: 10.1068/p5399

Toepper, M., Gebhardt, H., Bauer, E., Haberkamp, A., Beblo, T., Hallhofer, B., et al. (2014). The impact of age on load-related dorsolateral prefrontal cortex activation. Front. Aging Neurosci. 6:9. doi: 10.3389/fnagi.2014. 00009

Towes, J. N., and Neil, D. (1998). Analyzing human random generation behavior: a review of methods used and a computer program for describing performance. Behav. Res. Methods Instrum. Comput. 30, 583-591. doi: 10.3758/bf032 09475

Treisman, A. (1985). Preattentive processing in vision. Comput. Vis. Graph. Image Process. 31, 156-177. doi: 10.1016/s0734-189x(85)80004-9

Treisman, A. (1988). Features and objects: the fourteenth bartlett memorial lecture. Q. J. Exp. Psychol. A 40, 201-237. doi: 10.1080/027249888430 00104

Vangkilde, S., Coull, J. T., and Bundesen, C. (2012). Great expectations: temporal expectation modulates perceptual processing speed. J. Exp. Psychol. Hum. Percept. Perform. 38, 1183-1191. doi: 10.1037/a0026343

van der Linden, M., Beerten, A., and Pesenti, M. (1998). Age-related differences in random generation. Brain Cogn. 38, 1-16. doi: 10.1006/brcg.1997.0969

Vangkilde, S., Petersen, A., and Bundesen, C. (2013). Temporal expectancy in the context of a theory of visual attention. Philos. Trans. R. Soc. Lond. B Biol. Sci. 368:20130054. doi: 10.1098/rstb.2013.0054

van Leeuwen, S., Müller, N. G., and Melloni, L. (2009). Age effects on attentional blink performance in meditation. Conscious. Cogn. 18, 593-599. doi: 10.1016/j. concog.2009.05.001

Vaucher, P., Herzig, D., Cardodo, I., Herzog, M. H., Mangin, P., and Favrat, B. (2014). The trail making test as a screening instrument for driving performance in older drivers; a translational research. BMC Geriatr. 14:123. doi: 10.1186/1471-2318-14-123

Verhaeghen, P., and Cerella, J. (2002). Aging, executive control and attention: a review of meta-analyses. Neurosci. Biobehav. Rev. 26, 849-857. doi: 10.1016/s0149-7634(02)00071-4

Watson, D. G., and Maylor, E. (2002). Aging and visual marking: selective deficits for moving stimuli. Psychol. Aging 17, 321-339. doi: 10.1037/0882-7974.17. 2.321

Wilms, I. L., and Nielsen, S. (2014). Normative perceptual estimates for 91 healthy subjects age 60-75: impact of age, education, employment, physical exercise, alcohol and video gaming. Front. Psychol. 5:1137. doi: 10.3389/fpsyg.2014. 01137

Windsor, T. D., Anstey, K. J., Butterworth, P., Luszcz, M. A., and Andrews, G. R. (2007). The role of perceived control in explaining depressive symptoms associated with driving cessation in a longitudinal study. Gerontologist 47, 215-223. doi: 10.1093/geront/47.2.215

Wolfe, J. M. (1998). What can 1 million trials tell us about visual search? Psychol. Sci. 9, 33-39. doi: 10.1111/1467-9280.00006

Zacks, R. T., Hasher, L., and Li, K. Z. H. (2000). "Human memory," in The Handbook of Aging and Cognition, 2nd Edn. eds F. I. M. Craik and T. A. Salthouse (Mahwah, NJ: Lawrence Erlbaum Associates Publishers), 293-357.

Conflict of Interest Statement: The authors declare that the research was conducted in the absence of any commercial or financial relationships that could be construed as a potential conflict of interest.

The reviewer DW declared a collaboration with one of the authors $\mathrm{CH}$ to the handling Editor, who ensured that the process met the standards of a fair and objective review.

Copyright (C) 2017 Callaghan, Holland and Kessler. This is an open-access article distributed under the terms of the Creative Commons Attribution License (CC BY). The use, distribution and reproduction in other forums is permitted, provided the original author(s) or licensor are credited and that the original publication in this journal is cited, in accordance with accepted academic practice. No use, distribution or reproduction is permitted which does not comply with these terms. 